\title{
Exploring association between countries' self-reported International Health Regulations core capacity and COVID-19 control outcomes
}

\author{
Feng-Jen Tsai $^{1}$ (D, Chia-Ping Lin ${ }^{1}$, Battsetseg Turbat ${ }^{1}$ \\ ${ }^{1}$ Global Health and Health Security, College of Public Health, Taipei Medical University, Taipei, Taiwan \\ Keywords: human development index, health regulation, covid-19, universal health coverage \\ https://doi.org/10.29392/001c.21362
}

\section{Journal of Global Health Reports}

Vol. 5, 2021

\section{Background}

This study aimed to evaluate the association between countries' self-reported International Health Regulations (IHR) capacity and their COVID-19 control outcomes.

\begin{abstract}
Methods
Scores from the countries' 2019 self-assessment report (State Party Self-Assessment Annual Reporting, SPAR) were used to represent countries' core capacities. Two scales were used to represent countries' COVID-19 control outcomes: "governmental response to COVID-19," which included case public health (PH) measure intervals, case-PH measure speeds, government response stringency; and scale "COVID-19 outbreak progress within the country," which included days between the first global and domestic cases, number of case growing weeks, and percentage of case growing weeks between 01 January 31 May, 2020. One hundred and seventy-four countries with COVID-19 data were included in the analysis. The human development index (HDI), health workforce density (HWD), health expenditure (HE), and international travel volume (ITV) were collected as controls. Chi-square and logistic regression analyses were carried out.
\end{abstract}

\begin{abstract}
Results
Countries with high SPAR scores responded significantly earlier, but $49.46 \%$ of countries with high and very high HDI implemented public health measures within a month of the first global case, whereas $20 \%$ of countries implemented PH measures after there was already a domestic case. Compared with low ITV countries, countries with high ITV had a 15 times greater risk of early importation of COVID-19 cases and a greater risk of having more weeks over which case numbers increased. However, countries with low HE and SPAR scores as health service provision were at significantly higher risk of community outbreak escalation.
\end{abstract}

\section{Conclusions}

To some extent, countries' COVID-19 control outcomes were reflected by their SPAR scores. However, SPAR do not measure the effectiveness of communication mechanisms between health professionals and political leaders in the activation of response systems. The country-based health expertise and health system is crucial to limiting further viral spread in the community.

Emerging infectious diseases like coronavirus disease 2019 (COVID-19) pose significant health and security challenges for the world, damaging global economies, and public health. ${ }^{1-4}$ After the SARS pandemic in 2003, International Health Regulations (IHR) 2005 were adopted by the World Health Organization (WHO) to enhance global capacity to prevent and control infectious diseases. ${ }^{5}$ One of the approaches adopted by IHR 2005 was requiring member states to develop minimal core public health capacities for effective implementation.
To monitor progress in building these public health capacities, WHO introduced a self-assessment process for countries to report on their implementation of IHR 2005.6 In 2010, the IHR Secretariat at WHO developed the IHR Core Capacity Monitoring Framework and released the IHR Monitoring Tool (IHRMT) to monitor progress in implementing IHR core capacities. ${ }^{7}$ With this standardised data collection tool, it was recommended that countries fill out the IHRMT and submit completed reports to WHO annually. Because of insufficient attention to the self-assessment 
approach, WHO took the recommendation of review panels and adopted Joint External Evaluations (JEE) concerning national capacities in pandemic preparedness in $2016 .{ }^{8}$ To harmonise self and external evaluation tools, the IHRMT was updated to the IHR State Party self-assessment Annual Reporting (SPAR) tool in 2018. ${ }^{9}$

The purpose of monitoring countries' self-assessment progress is to evaluate their preparedness for infectious diseases like the current COVID-19 pandemic. While countries are obligated to develop their capacity for infectious disease control and report the outcome annually to the WHO, this effort should provide information about countries' effectiveness in responding to pandemics in a timely manner. In theory, higher SPAR scores indicate better preparedness; therefore, countries with higher SPAR scores should perform better in their responses to a pandemic like COVID-19.

Previous studies showed that countries' self-reported IHRMT scores were significantly associated with their infectious disease control outcomes, although some countries unreasonably gave themselves full scores. ${ }^{10}$ However, studies focusing on associations between countries' SPAR scores and their COVID-19 control outcomes have not yet been reported.

In the early stages of the COVID-19 outbreak, studies based on SPAR showed that countries' capacities to prevent, detect, and respond to outbreaks are variable. ${ }^{11}$ However, half of the countries should have been capable of responding effectively to a pandemic like COVID-19. The Prevent Epidemics organisation performed a simple analysis, presented as the ReadyScore, about countries' effectiveness in responding to COVID-19. They found that better prepared countries "do a better job finding cases and preventing deaths.” However, "better prepared countries did not act sooner to implement PHSMs (public health and social measures)". ${ }^{12}$ Both studies were preliminary and recommended additional studies to understand national preparedness capacity in relation to COVID-19. Therefore, we conducted this study to understand the association between countries' self-reported IHR capacity scores and their COVID-19 control outcomes.

\section{METHODS}

\section{STUDY DESIGN}

For formation of the following outcomes, we downloaded the COVID-19 data from large-scale dataset collected from 'Our World in Data' websites, including the daily COVID-19 cases for each country and their implemented public health $(\mathrm{PH})$ measures, which are from the European Centre for Disease Prevention and Control (ECDC) and the Oxford COVID-19 Government Response Tracker (OxCGRT), respectively. ${ }^{13}$ Information from 174 countries was found in the merged dataset, and analysed in the study. We chose the duration 1 January to 31 May 2020 because the world first knew about COVID-19 in December 2019, and the end of May 2020 could be considered the end of the first wave of the global outbreak. ${ }^{14}$ Additionally, we used a cross-sectional design for the country-based analysis and estimated the odds ratio between countries' self-assessment capacity scores and COVID-19 control outcomes.

\section{SPAR (IHR STATE PARTY SELF-ASSESSMENT ANNUAL REPORTING TOOL)}

We used 2019 SPAR scores to represent countries' self-evaluated core capacity. SPAR is a questionnaire to monitor countries' progress in implementing IHR guidelines. ${ }^{15}$ The questionnaire consists of 13 sections including core capacities for infectious diseases controls (seven items), point of entry (POE), health service provision (HSP), and four other hazards, as identified and delineated by WHO to match the obligations outlined in Annex 1 of the IHR. The seven core capacities for infectious disease control include legislation and financing, IHR coordination and national IHR focal point functions, laboratory, surveillance, human resources, national health emergency frameworks (combination of the previous IHRMT items response and preparedness), and risk communication. The four hazards include zoonotic events and the human-animal interface, food safety, chemical events, and radiation emergencies.

Countries' SPAR responses comprise percentages of implementation ranging from 0 to 100 . We downloaded 2019 SPAR scores from the WHO website on July 15, 2020. Selfreported SPAR scores from 174 countries were available, and these were used in the study.

Average scores of the seven core capacities were calculated to represent national capacities for infectious disease control. The average scores of the seven core capacities plus point of entry were calculated to include countries' capacity for managing airports, ports, and ground crossings, which represent countries' overall capacity for preventing infectious disease spread from other countries. Then both average SPAR core capacity scores were classified into three groups-high, medium and low for analysis. Countries with scores in the upper tertile were defined as high-SPAR scores group; others were defined as countries with medium and low SPAR scores.

Additionally, we added the SPAR item health service provision to evaluate countries' capacity of "access to essential health services” in 2018. Because this item could represent medical countermeasures and personnel deployment for providing health services to confirm and treat infected cases, we also took it as a country's back-end system indicator.

\section{INFECTIOUS DISEASE CONTROL OUTCOMES}

Based on the rationale that early detection and response is fundamental in infectious disease control to avoid escalation of a pandemic, we used two scales to represent countries' COVID-19 response and control outcome: "governmental response to COVID-19" and “COVID-19 outbreak progress within the country."

\section{GOVERNMENTAL RESPONSE TO COVID-19}

The scale of the governmental response to COVID-19 measures the speeds and the stringency of countries' responses to COVID-19 after awareness of the first global case listed on the WHO website on December 31, 2019.16 The rationale for taking the governmental response to COVID-19 as an outcome is that countries' earlier and stricter implemen- 
tation of $\mathrm{PH}$ measures might represent countries' better awareness of and vigilance regarding infectious disease control.

The governmental response to COVID-19 included three indicators: case-PH measure intervals, case-PH measure speeds, and government response stringency. Detailed information about these three indicators is described below.

CASE-PH MEASURE INTERVALS AND SPEEDS

The case-PH measure intervals and speeds are indicators of countries' response time after awareness of the first global and domestic cases in their implementation of $\mathrm{PH}$ measures for preventing further spread of the disease. We used the gap in days between knowledge of the first global case and countries' first PH measures to indicate the case-PH measure intervals. We then divided the case- $\mathrm{PH}$ measure intervals into two groups: "favorable" and "unfavorable," based on their averages.

We further developed the indicator case-PH measure speeds by countries' response statuses and classified countries into three groups by their response speeds: favorable, moderate and unfavorable. If countries implemented $\mathrm{PH}$ measures before the first domestic case and even before 31 January 2020, they were categorised as favorable. If they implemented $\mathrm{PH}$ measures after the first global case but before the first domestic case and after 31 January 2020, they were categorised as moderate. Countries that implemented $\mathrm{PH}$ measures after their first domestic case were classified as unfavourable.

\section{GOVERNMENT RESPONSE STRINGENCY}

We used the "Government response stringency Index" in the dataset of Our World in Data in this study. ${ }^{17}$ The index is a composite measure based on nine response indicators including school closures, workplace closures, and travel bans, rescaled to a value from 0 to 100 (100 being the strictest) to represent the stringencies of countries' responses to COVID-19. The details of the methodologies are described on the website. Specifically, we used the index from the third day after discovery of the first domestic case as the indicator. We classified the index values into two groups, strong and weak using the average as cut-off point for analysis.

\section{COVID-19 OUTBREAK PROGRESS WITHIN THE COUNTRY}

The scale of COVID-19 outbreak progress measures countries' outcomes in preventing the importation of COVID-19 from other countries and the further spread of the disease within the country. COVID-19 outbreaks progress within the country includes the following indicators: days between the first global and domestic cases, number of case growing weeks, and percentage of case growing weeks between 1 January and 31 May 2020.

\section{DAYS BETWEEN FIRST GLOBAL AND DOMESTIC CASES}

Although the days between the first global and domestic cases might represent countries' efforts in the prevention of importation of the virus from abroad, we used it as one of the outcome indicators. If countries effectively prevented importation of the COVID-19 virus from other countries, the numbers of days between the first global and domestic cases would be longer. We further classified the numbers of days between the first global and domestic cases into two groups, favorable and unfavorable using the average as the cut-off point for analysis.

NUMBER AND PERCENTAGE OF WEEKS OVER WHICH CASE COUNTS INCREASED BETWEEN JANUARY AND MAY 2020

The rationale was that if a week's case growth rate was positive compared with the previous week, the outbreak in the country was leveling up over that week. Therefore, we used the number of weeks with positive case growth rates between 1 January and 31 May 2020 to represent the countries' outbreak control outcomes.

We used the item "weekly growth rate" from 'Our World in Data' to calculate the "number of case growing weeks" between 1 January and 31 May 2020. ${ }^{18}$ The definition of "weekly growth rate" used in the same database to form the indicator was the percentage change in the number of new confirmed cases over the number in the last seven days relative to the previous seven days. We used the weekly growth rate of the last day of the week for the indicator. Furthermore, we calculated the percentage of weeks with positive case growth rates during the period to represent domestic control outcomes after having cases within a country. Both the "number of case growing weeks" and "percentage of case growing weeks" between 1 January and 31 May 2020 were divided into two groups by using averages as cut-off point for further analysis.

\section{MEASUREMENTS}

With the rationale that national infectious disease control capacity includes systematic elements like legislation, health investment and coordination, and human resources in the form of trained medical professionals, ${ }^{8,19}$ we searched the Human Development Index (HDI) from the United Nations Development Program (UNDP) and information from WHO regarding the density of physicians and nurses (health workforce density, HWD) and total health expenditure (HE) to represent the general health capacity of a country. ${ }^{20-22}$

By definition, human development encompasses three dimensions: life expectancy, which indicates population health and longevity; education, which indicates level of knowledge and education; and per capita income, which indicates purchasing power parity. Using indicators mainly collected from official statistics, HDI was calculated as the simple mean of dimensional indices ranging from 0 to 1 , with 1 representing the highest degree of human development and 0 the lowest. The details of the methodology were described in the technical notes section of the report. ${ }^{21}$ We used the 2020 HDI (2019 data) to represent the human development status of each country in that year. In addition, the categories used by the HDI, i.e., very high, high, medium, and low development countries, were also used in the study.

Information about each country's density of physicians and nurses was collected from WHO websites. ${ }^{23}$ Then the sum of these two scores was calculated and used as the in- 
dex of HWD in the study. We then categorised countries into high and low health workforce countries based on the sum of the density of physicians and nurses in each country. Countries with high and low HWD values were defined as countries with large and small health workforces, respectively.

Information about each country's total HE was also collected from WHO websites to represent their national investment in health. We then categorised countries into two groups by cut-off point as average.

While the frequency of international travel increases the risk of COVID-19 outbreaks, we also collected information from the World Bank regarding the number of each country's international tourists in 2018 to represent the risk of importing the virus. ${ }^{24}$ The World Bank classifies the number of arrivals of international tourists into five levels (level 5 is the highest). Although two-thirds of countries were classified as level 1 to 2, we reclassified countries into two international travel groups, high and low using the cut-off point at the upper bound of level 2 .

\section{STATISTICAL ANALYSIS}

The Chi-square test was first applied to compare countries' HDI, HWD, HE, ITV, and SPAR scores between different COVID-19 response and control outcomes. Then binary regression was applied to understand the relationship between countries' HDI, HWD, HE, ITV, and SPAR scores and their responses, including case-PH measure intervals, casePH measure speeds, government response stringency, days between global and domestic first case, number of case growing weeks, and percentage of case growing weeks. Each factor that was determined to be statistically different between groups by Chi-square analysis was included in the model. Multivariate logistic regression was then applied for evaluating the risks between response and control outcomes of the countries and their HDI, HWD, HE, ITV, and SPAR scores. All analyses were performed using SPSS, Version 18.0 (IBM, Armonk, NY, USA). P<0.05 was statistically significant.

\section{ROLE OF FUNDING SOURCE}

The funding source of this study had no role in the study design, data collection, data analysis, data interpretation, or writing of the report. The corresponding author had full access to all the data in the study and had final responsibility for the decision to submit it for publication.

\section{RESULTS}

Among the 174 countries with COVID-19 data, the average number of days from the first global case to the first $\mathrm{PH}$ measures (case-PH measure intervals) was 42.3 . The average number of days from the first global to the first domestic case was 64.88 . The average number of case growing weeks and the percentage of case growing weeks between Jan. 1 and May 31, 2020 were 6.06 and 56.44\%, respectively.

Furthermore, the average number of days from the first global to the first domestic case in very high, high, medium, and low HDI countries was 51.11 days, 65.93 days, 66.97 days and 81.78 days, respectively. The average number of case growing weeks of very high, high, medium, and low HDI countries was 6.81 weeks, 6.22 weeks, 6.75 weeks, and 5 weeks, respectively. The average percentage of case growing weeks in very high, high, medium, and low HDI countries was $51.93 \%, 56.08 \%, 66.42 \%$, and $58.75 \%$, respectively.

Among the 147 countries with SPAR scores, the average score of the seven core capacities items was 67.5, ranged from 17.14 to 99 . Surveillance, laboratory and IHR coordination, and national IHR focal point functions had the highest scores. Risk communication, national health emergency, and human resources had the lowest scores. Point of entry had the lowest score of all the items, in addition to the seven core capacities.

\section{COMPARISON OF HDI, HWD, HE ITV AND SPAR SCORES BETWEEN DIFFERENT GOVERNMENTAL RESPONSE TO COVID-19 OUTCOME GROUPS BY CHI-SQUARE \\ ANALYSIS}

Comparison of HDI, HWD, HE, ITV, and SPAR scores between different governmental responses to COVID-19 indicator groups by Chi-square analysis is shown in Table $\mathbf{1}$. HDI, HWD, ITV, and SPAR scores were significantly different between indicator groups.

Indicator as "case PH measure intervals," HWD, and SPAR scores, including the seven core capacity scores, seven core capacity plus POE scores, and HSP were significantly different between groups. In the unfavorable group, the percentage of countries with low HWD and low SPAR scores was significantly higher.

Indicator as "case PH measure speeds”, HDI, HWD, ITV, and SPAR scores were significantly different between groups. The percentage of countries with very high and high HDI statuses was significantly higher in both the favorable and unfavorable groups. Of the countries with very high and high HDI statuses, $49.46 \%$ took PH measures within a month after the first global first was documented, whereas $20 \%$ of these countries acted late; they took PH measures only after they already had a domestic case. Similarly, the percentage of countries with high ITV was significantly higher in both favorable and unfavorable countries, but the percentage of countries with high HWD was only significantly higher in the favorable group.

For government response stringency, all variables were significantly different between groups. Significantly more countries with high HDI statuses, HWD, HE, ITV, and high SPAR scores implemented stricter public health measures after identifying their first domestic case.

The percentages of countries with high SPAR scores including the seven core capacity average scores, seven core capacity plus POE average scores, and HSP scores were all significantly higher in both groups of countries.

COMPARISON OF HDI, HWD, HE, ITV AND SPAR SCORES BETWEEN DIFFERENT COVID-19 OUTBREAK PROGRESS WITHIN THE COUNTRY INDICATOR GROUPS BY CHISQUARE ANALYSIS

HDI, ITV, HWD, HE, and SPAR scores between different COVID-19 outbreak progress was compared within the 
country outcome groups by Chi-square analysis (Table 2). tries in terms of their COVID-19 outbreak progress within Above variables were significantly different between coun- their indicator groups. 
Table 1. Governmental response to COVID-19

\begin{tabular}{|c|c|c|c|c|c|c|c|c|c|c|}
\hline & \multicolumn{3}{|c|}{ case-PH measure intervals } & \multicolumn{4}{|c|}{ case-PH measure speeds } & \multicolumn{3}{|c|}{ government response stringency } \\
\hline & $\begin{array}{c}\text { Favorable } \\
(\mathrm{n}=99)\end{array}$ & $\begin{array}{c}\text { Unfavorable } \\
(n=73)\end{array}$ & P-value & $\begin{array}{c}\text { Favorable } \\
(\mathrm{n}=69)\end{array}$ & $\begin{array}{c}\text { Moderate } \\
(n=74)\end{array}$ & $\begin{array}{c}\text { Unfavorable } \\
(\mathrm{n}=29)\end{array}$ & P-valuet & $\begin{array}{l}\text { Strong } \\
(n=75)\end{array}$ & $\begin{array}{l}\text { Weak } \\
(n=97)\end{array}$ & P-value \\
\hline \multicolumn{11}{|l|}{ HDI } \\
\hline Very High & $36(39.6 \%)$ & $17(25.8 \%)$ & $0.051^{*}$ & $27(39.7 \%)$ & $14(22.6 \%)$ & $12(44.4 \%)$ & $0.036^{* *}$ & $8(12.3 \%)$ & $45(48.9 \%)$ & $<0.01^{* * *}$ \\
\hline High & $24(26.4 \%)$ & $16(24.2 \%)$ & & $19(27.9 \%)$ & $14(22.6 \%)$ & $7(25.9 \%)$ & & $19(29.2 \%)$ & $21(22.8 \%)$ & \\
\hline Medium & $19(20.9 \%)$ & $13(19.7 \%)$ & & $14(20.6 \%)$ & $13(21.0 \%)$ & $5(18.5 \%)$ & & $17(26.2 \%)$ & $15(16.3 \%)$ & \\
\hline low & 12 (13.2\%) & 20 (30.3\%) & & $8(11.8 \%)$ & 21 (33.9\%) & $3(11.1 \%)$ & & $21(32.3 \%)$ & $11(12.0 \%)$ & \\
\hline \multicolumn{11}{|c|}{ International travel volume } \\
\hline High & $62(73.8 \%)$ & 34 (64.2\%) & 0.229 & $46(73.0 \%)$ & $27(57.4 \%)$ & $23(85.2 \%)$ & $0.034^{* *}$ & $22(41.5 \%)$ & $74(88.1 \%)$ & $<0.01^{* * *}$ \\
\hline Low & $22(26.2 \%)$ & $19(35.8 \%)$ & & $17(27.0 \%)$ & $20(42.6 \%)$ & $4(14.8 \%)$ & & $31(58.5 \%)$ & $10(11.9 \%)$ & \\
\hline \multicolumn{11}{|c|}{ Health workforce density } \\
\hline High & $51(60.0 \%)$ & $23(36.5 \%)$ & $<0.01^{* * *}$ & 40 (63.5\%) & $23(39.7 \%)$ & $11(40.7 \%)$ & $0.018^{* *}$ & 20 (34.5\%) & $54(60.0 \%)$ & $<0.01^{* * *}$ \\
\hline Low & $34(40.0 \%)$ & 40 (63.5\%) & & $23(36.5 \%)$ & $35(60.3 \%)$ & $16(59.3 \%)$ & & $38(65.5 \%)$ & $36(40.0 \%)$ & \\
\hline \multicolumn{11}{|l|}{ Health expenditure } \\
\hline High & $46(50.0 \%)$ & $29(45.3 \%)$ & 0.564 & $33(47.8 \%)$ & $29(49.2 \%)$ & $13(46.4 \%)$ & 0.971 & $25(39.7 \%)$ & $50(53.8 \%)$ & $0.084^{*}$ \\
\hline Low & $46(50.0 \%)$ & $35(54.7 \%)$ & & $36(52.2 \%)$ & $30(50.8 \%)$ & $15(53.6 \%)$ & & $38(60.3 \%)$ & $43(46.2 \%)$ & \\
\hline \multicolumn{11}{|l|}{ SPAR scores (2019) } \\
\hline \multicolumn{11}{|c|}{7 core capacity average scores } \\
\hline High & $36(42.4 \%)$ & $15(24.6 \%)$ & $0.044^{* *}$ & $27(43.5 \%)$ & $11(19.0 \%)$ & $13(50.0 \%)$ & $<0.01^{* * *}$ & $13(22.0 \%)$ & $38(43.7 \%)$ & $0.020^{* *}$ \\
\hline Medium & $27(31.8 \%)$ & $20(32.8 \%)$ & & $21(33.9 \%)$ & $20(34.5 \%)$ & $6(23.1 \%)$ & & $21(35.6 \%)$ & $26(29.9 \%)$ & \\
\hline Low & $22(25.9 \%)$ & $26(42.6 \%)$ & & $14(22.6 \%)$ & $27(46.6 \%)$ & $7(26.9 \%)$ & & $25(42.4 \%)$ & $23(26.4 \%)$ & \\
\hline \multicolumn{11}{|c|}{7 core capacity plus POE average scores } \\
\hline High & $36(43.4 \%)$ & $14(23.0 \%)$ & $0.023^{* *}$ & $27(45.0 \%)$ & $12(20.7 \%)$ & $11(42.3 \%)$ & $0.022^{* *}$ & $10(17.2 \%)$ & $40(46.5 \%)$ & $<0.01^{* * *}$ \\
\hline Medium & $26(31.3 \%)$ & $21(34.4 \%)$ & & $20(33.3 \%)$ & $19(32.8 \%)$ & $8(30.8 \%)$ & & 24 (41.4\%) & $23(26.7 \%)$ & \\
\hline Low & $21(25.3 \%)$ & $26(42.6 \%)$ & & $13(21.7 \%)$ & $27(46.6 \%)$ & $7(26.9 \%)$ & & $24(41.4 \%)$ & $23(26.7 \%)$ & \\
\hline \multicolumn{11}{|c|}{ Health service provision } \\
\hline High & $53(62.4 \%)$ & $28(45.9 \%)$ & $0.049^{* *}$ & $39(62.9 \%)$ & $23(39.7 \%)$ & $19(73.1 \%)$ & $<0.01^{* * *}$ & $23(39.0 \%)$ & $58(66.7 \%)$ & $<0.01^{* * *}$ \\
\hline Low & $32(37.6 \%)$ & $33(54.1 \%)$ & & $23(37.1 \%)$ & 35 (60.3\%) & $7(26.9 \%)$ & & 36 (61.0\%) & $29(66.7 \%)$ & \\
\hline
\end{tabular}

HDI - Human Development Index, POE - Point of Entry, SPAR - State Party Self-Assessment Annual Reporting

All data are $\mathrm{n}(\%)$ or P-value. Significant level: $\mathrm{p}<0.1^{* *}, \mathrm{p}<0.05^{* * *}, \mathrm{p}<0.01^{* * * * *}$

†Comparison of HDI, international travel volume, health workforce density, health expenditure and SPAR scores between different governmental response to COVID-19 outcome groups by Chi-square analysis. 
Table 2. COVID-19 outbreak progress within the country

\begin{tabular}{|c|c|c|c|c|c|c|c|c|c|}
\hline & \multicolumn{3}{|c|}{ days between the first global and domestic cases } & \multicolumn{3}{|c|}{ number of case growing weeks $\ddagger$} & \multicolumn{3}{|c|}{ percentage of case growing weeks $¥$} \\
\hline & $\begin{array}{c}\text { Favorable } \\
(\mathrm{n}=72)\end{array}$ & $\begin{array}{l}\text { Unfavorable } \\
(n=100)\end{array}$ & P-value & $\begin{array}{c}\text { Favorable } \\
(\mathrm{n}=100)\end{array}$ & $\begin{array}{c}\text { Unfavorable } \\
(\mathrm{n}=74)\end{array}$ & P-value $†$ & $\begin{array}{l}\text { Strong } \\
(\mathrm{n}=92)\end{array}$ & $\begin{array}{l}\text { Weak } \\
(n=77)\end{array}$ & P-value \\
\hline \multicolumn{10}{|l|}{ HDI } \\
\hline Very High & $12(13.5 \%)$ & $41(60.3 \%)$ & $<0.01^{* * *}$ & $25(28.7 \%)$ & $28(38.9 \%)$ & $0.02^{* *}$ & $35(42.7 \%)$ & $17(23.0 \%)$ & $0.061^{*}$ \\
\hline High & $26(29.2 \%)$ & $14(20.6 \%)$ & & $20(23.0 \%)$ & $21(29.2 \%)$ & & $18(22.0 \%)$ & $22(29.7 \%)$ & \\
\hline Medium & $22(24.7 \%)$ & $10(14.7 \%)$ & & $16(18.4 \%)$ & $16(22.2 \%)$ & & $13(15.9 \%)$ & $19(25.7 \%)$ & \\
\hline low & $29(32.6 \%)$ & $3(3.3 \%)$ & & $26(29.9 \%)$ & $7(9.7 \%)$ & & $16(19.5 \%)$ & $16(21.6 \%)$ & \\
\hline \multicolumn{10}{|c|}{ International travel volume } \\
\hline High & $33(46.5 \%)$ & $63(95.5 \%)$ & $<0.01^{* * *}$ & $43(53.8 \%)$ & $53(91.4 \%)$ & $<0.01^{* * *}$ & $49(64.5 \%)$ & $45(77.6 \%)$ & $0.10^{*}$ \\
\hline Low & $38(53.5 \%)$ & $3(4.5 \%)$ & & $37(46.3 \%)$ & $5(8.6 \%)$ & & $27(35.5 \%)$ & $13(22.4 \%)$ & \\
\hline \multicolumn{10}{|c|}{ Health workforce density } \\
\hline High & $27(32.9 \%)$ & $47(71.2 \%)$ & $<0.01^{* * *}$ & $39(47.6 \%)$ & $36(52.9 \%)$ & 0.512 & $47(61.8 \%)$ & $27(38.0 \%)$ & $<0.01^{* * *}$ \\
\hline Low & $55(67.1 \%)$ & $19(28.8 \%)$ & & $43(52.4 \%)$ & $32(47.1 \%)$ & & $29(38.2 \%)$ & $44(62.0 \%)$ & \\
\hline \multicolumn{10}{|l|}{ Health expenditure } \\
\hline High & $38(43.7 \%)$ & $37(53.6 \%)$ & 0.217 & $46(53.5 \%)$ & $30(41.7 \%)$ & 0.139 & $47(57.3 \%)$ & $27(37.0 \%)$ & $0.011^{* *}$ \\
\hline Low & 49 (56.3\%) & $32(46.4 \%)$ & & $40(46.5 \%)$ & $42(58.3 \%)$ & & $35(42.7 \%)$ & $46(63.0 \%)$ & \\
\hline \multicolumn{10}{|l|}{ SPAR scores (2019) } \\
\hline \multicolumn{10}{|c|}{7 core capacity average scores } \\
\hline High & $13(15.5 \%)$ & $38(61.3 \%)$ & $<0.01^{* * *}$ & $20(25.3 \%)$ & $31(45.6 \%)$ & $<0.01^{* * *}$ & $27(37.0 \%)$ & $24(33.3 \%)$ & 0.826 \\
\hline Medium & $33(39.3 \%)$ & $14(22.6 \%)$ & & $24(30.4 \%)$ & $24(35.3 \%)$ & & $22(30.1 \%)$ & $25(34.7 \%)$ & \\
\hline Low & $38(45.2 \%)$ & $10(16.1 \%)$ & & $35(44.3 \%)$ & $13(19.1 \%)$ & & $24(32.9 \%)$ & $23(31.9 \%)$ & \\
\hline \multicolumn{10}{|c|}{7 core capacity plus POE average scores } \\
\hline High & $13(15.9 \%)$ & $37(59.7 \%)$ & $<0.01^{* * *}$ & $21(26.6 \%)$ & $29(43.9 \%)$ & $<0.01^{* * *}$ & $29(39.7 \%)$ & $21(30.0 \%)$ & 0.417 \\
\hline Medium & 32 (39.0\%) & $15(24.2 \%)$ & & $24(30.4 \%)$ & $24(36.4 \%)$ & & $21(28.8 \%)$ & $26(37.1 \%)$ & \\
\hline Low & $37(45.1 \%)$ & $10(16.1 \%)$ & & $34(43.0 \%)$ & $13(19.7 \%)$ & & $23(31.5 \%)$ & $23(32.9 \%)$ & \\
\hline \multicolumn{10}{|c|}{ Health service provision } \\
\hline High & $33(39.3 \%)$ & $48(77.4 \%)$ & $<0.01^{* * *}$ & $40(50.6 \%)$ & $42(61.8 \%)$ & 0.175 & $48(65.8 \%)$ & $33(45.8 \%)$ & $0.016^{* *}$ \\
\hline Low & $51(60.7 \%)$ & $14(22.6 \%)$ & & $39(49.4 \%)$ & $26(38.2 \%)$ & & $25(34.2 \%)$ & $39(54.2 \%)$ & \\
\hline
\end{tabular}


Exploring association between countries' self-reported International Health Regulations core capacity and COVID-19 control..

HDI - Human Development Index, POE - Point of Entry, SPAR - State Party Self-Assessment Annual Reporting

All data are $\mathrm{n}(\%)$ or P-value. Significant level: $\mathrm{p}<0.1 \%, \mathrm{p}<0.05^{* * *}, \mathrm{p}<0.01^{* * * *}$

†Comparison of HDI, international travel volume, health workforce density, health expenditure and SPAR scores between different COVID-19 outbreak progress within the country outcome groups by Chi-square analysis.

† Number of case growing weeks and percentage of case growing weeks are between Jan. 1 and May 31, 2020. 
Indicator such as "days between the first global and domestic cases," HDI, HWD and ITV were significantly different between groups. Of the countries with very high and high HDI statuses, $81 \%$ were placed in the unfavorable group, whereas $58 \%$ of medium and low HDI countries were placed in the favorable group. Also, $95 \%$ of countries with high ITV were placed in unfavorable group, whereas $54 \%$ of countries with low ITV were placed in the favorable group. Similarly, there were $71 \%$ countries with high workforce density in unfavorable group, whereas there were 67\% countries with low HWD in the favorable group. Furthermore, SPAR scores including seven core capacity average scores, seven core capacity plus POE averages, and HSP were significantly different between groups. More than $60 \%$ of the countries in the unfavorable group had high average seven core capacity scores, seven core capacity plus POE average scores, and HSP scores, which was significantly more than those in the favorable group.

Indicator as "number of case growing weeks," HDI and ITVs were significantly different between groups. The percentage of countries with very high and high HDI statuses, and high ITV was significantly higher in the unfavorable group. Similarly, the percentage of countries with high average seven core capacity scores, and seven core capacity with POE scores was significantly higher in the unfavorable group.

For indicator as "percentage of case growing weeks," was different with other two indicators, HWD, HE and SPAR scores as HSP were the factors significantly different between groups. There was a significantly greater percentage of countries with high HWD and HE in the favorable group than in the unfavorable group.

ASSOCIATION BETWEEN HDI, HWD, HE, ITV, AND SPAR SCORES BETWEEN GOVERNMENTAL RESPONSE TO COVID-19 AND COVID-19 OUTBREAK PROGRESS WITHIN THE COUNTRY INDICATORS

Associations between HDI, HWD, HE, ITV, and SPAR scores between governmental responses to COVID-19 and COVID-19 outbreak progress within the country by regression (Table 3).

Governmental responses to COVID-19, countries' case$\mathrm{PH}$ measure intervals, case-PH measure speeds, and government response stringency were significantly associated with their HDI, HWD, ITV, and SPAR scores. Countries with medium SPAR scores based on the seven core capacities plus POE had significantly higher risks of implementing PH measures later than countries with high SPAR scores. In the unfavorable-response speed group, countries with the medium HDI status had significantly lower risks of responding late, and countries with low HWD had eight times the risk of responding late. In terms of the stringencies of governmental responses, countries with the low HDI status had significantly lower risks of implementing lax $\mathrm{PH}$ measures; countries with high ITV had three times the risk of implementing lax PH measures; and countries with low seven core capacity plus POE scores had 10 times the risk of implementing lax $\mathrm{PH}$ measures.

After controlling for confounders, COVID-19 outbreak progress within the country including days between the first global and domestic cases and the number and percentage of case growing weeks are significantly associated with HE, ITV, and SPAR scores. Countries with high ITV were 15 times more likely than countries with low ITV to have more days between the first global and domestic cases. Similarly, countries with high ITV had a significantly higher risk of having higher number of case growing weeks than countries with low ITV $(\mathrm{OR}=5.867)$. Because imported COVID-19 cases occurred much later, countries with low SPAR scores had significantly lower risks of having higher numbers of case growing weeks.

In contrast, countries with low total HE and low HSP scores had significantly higher risks of having higher percentages of case growing weeks. In detail, countries with low total HE had twice the risk of having higher percentage of case growing weeks than countries with high total HE. Countries with low HSP scores had four times the risk of having higher percentage of case growing weeks than countries with high HSP scores.

\section{DISCUSSION}

This is the first study that focused on the association between countries' self-reported national core capacity regarding infectious disease regulated by IHR (SPAR) with their COVID-19 control outcomes. There are significantly more countries with high health workforce density and high SPAR scores that took PH measures early. However, countries with very high and high HDI statuses, and high SPAR scores performed very differently, which resulted in the relationship of countries' HDI statuses and their response speed statuses forming a U shape. Of the countries with very high and high HDI statuses, $49.46 \%$ took $\mathrm{PH}$ measures within a month after the first global case, whereas 20\% acted late because they took $\mathrm{PH}$ measures after there were already domestic cases. Moreover, countries with high ITV had a 15 times higher risk of importing COVID-19 cases earlier and five times the risk of having a greater number of case growing weeks than low ITV countries. In contrast, countries with low HE and low HSP had significantly higher risk of having higher percentage of case growing weeks.

The study results showed that the speeds of implementing $\mathrm{PH}$ measures differed significantly between countries with very high and high HDI statuses, which was echoed in the comment that "science is one thing, leadership is another". ${ }^{25-27}$ Even in countries with high health security capacities, the system could not be activated without political commitment. The leaders of such countries usually rejected proposals for implementing public health measures due to concerns about their economic impact. Although there have already been warnings about further health impacts because of economic recession brought on by COVID-19, leaders' struggles between health and economics are understandable. ${ }^{28}$ However, a recent study found that "countries that have managed to protect their population's health in the pandemic have generally also protected their economy," which is the opposite result, assuming that leaders have to face trade-offs between health and economics. ${ }^{29}$ Therefore, this finding suggests that communication about infectious disease control between professionals and politicians should be strengthened. 
Table 3. Risk of COVID-19 response and control outcomes

\begin{tabular}{|c|c|c|c|c|c|c|c|}
\hline & \multicolumn{3}{|c|}{ Governmental response to COVID-19† } & \multicolumn{4}{|c|}{ COVID-19 outbreak progress within the country $\dagger$} \\
\hline & $\begin{array}{l}\text { case-PH measure } \\
\text { intervals }\end{array}$ & $\begin{array}{c}\text { case-PH measure } \\
\text { speeds } \\
\text { (Favorable vs } \\
\text { Moderate) }\end{array}$ & $\begin{array}{c}\text { case-PH measure } \\
\text { speeds } \\
\text { (Favorable vs } \\
\text { Unfavorable) }\end{array}$ & $\begin{array}{l}\text { government response } \\
\text { stringency }\end{array}$ & $\begin{array}{l}\text { days between the } \\
\text { first global and } \\
\text { domestic cases }\end{array}$ & $\begin{array}{l}\text { number of case } \\
\text { growing weeks }\end{array}$ & $\begin{array}{r}\text { percentage of g } \\
\text { weeks }\end{array}$ \\
\hline \multicolumn{8}{|l|}{ HDI } \\
\hline Very High & 1 (ref) & 1 (ref) & 1 (ref) & 1 (ref) & 1 (ref) & 1 (ref) & 1 (ref) \\
\hline High & $0.706(0.229-2.179)$ & $0.841(0.225-3.145)$ & $0.308(0.061-1.569)$ & $0.367(0.085-1.591)$ & $0.389(0.106-1.425)$ & $1.264(0.374-4.278)$ & $1.079(0.348-3.3$ \\
\hline Medium & $0.434(0.89-2.120)$ & $0.358(0.044-2.929)$ & $0.120 *(0.013-1.121)$ & $0.350(0.048-2.573)$ & $0.875(0.126-6.055)$ & $1.302(0.208-8.143)$ & $0.804(0.151-4.3$ \\
\hline low & $0.834(0.138-5.039)$ & $1.629(0.107-24.706)$ & $0.198(0.008-4.737)$ & $0.014^{* * *}(0.001-0.341)$ & 0 & $0.970(0.066-14.346)$ & $0.350(0.051-2.3$ \\
\hline \multicolumn{8}{|c|}{ International travel volume } \\
\hline Low &.. & 1 (ref) & 1 (ref) & 1 (ref) & 1 (ref) & 1 (ref) &.. \\
\hline High &.. & $0.848(0.228-3.149)$ & $2.761(0.485-15.706)$ & $3.596 *(0.969-13.345)$ & $15.492^{* * *}(2.387-100.533)$ & $5.867^{* *}(1.471-23.394)$ &.. \\
\hline \multicolumn{8}{|c|}{ Health workforce density } \\
\hline High & 1 (ref) & 1 (ref) & 1 (ref) & 1 (ref) & 1 (ref) & 1 (ref) & 1 (ref) \\
\hline Low & $1.728(0.532-5.608)$ & $2.129(0.450-10.069)$ & $8.303^{* *}(1.475-46.729)$ & $0.727(0.176-3.007)$ & $0.435(0.107-1.772)$ & $0.959(0.253-3.641)$ & $2.224(0.629-7 . \varepsilon$ \\
\hline \multicolumn{8}{|l|}{ Health expenditure } \\
\hline High &. &.. &. &.. &. & .. & 1 \\
\hline Low &.. &.. &. &.. &.. &.. & $2.656^{* *}(1.171-\epsilon$ \\
\hline \multicolumn{8}{|l|}{ SPAR scores (2019) } \\
\hline \multicolumn{8}{|c|}{7 core capacity average scores } \\
\hline High &. &. &. &. & 1 (ref) &. &. \\
\hline Medium & .. & .. &.. &.. & $0.187^{* * *}(0.057-0.617)$ &.. &.. \\
\hline Low &.. &.. &.. &.. & $1.350(0.157-11.602)$ &.. &.. \\
\hline \multicolumn{8}{|c|}{7 core capacity plus POE average scores } \\
\hline High & 1 (ref) & 1 (ref) & 1 (ref) & 1 (ref) &. & 1 (ref) & 1 (ref) \\
\hline Medium & $2.535^{*}(0.910-7.058)$ & $1.726(0.547-5.442)$ & $1.501(0.427-5.277)$ & $0.620(0.184-2.083)$ &.. & $0.920(0.319-2.650)$ & $1.074(0.389-2.9$ \\
\hline Low & $2.79(0.637-12.226)$ & $3.73(0.669-20.808)$ & $3.467(0.490-27.126)$ & $10.963^{*}(0.783-153.589)$ &. & $0.073^{* *}(0.009-0.622)$ & $0.398(0.074-2.1$ \\
\hline \multicolumn{8}{|c|}{ Health service provision } \\
\hline High & 1 (ref) &.. &.. & 1 (ref) & 1 (ref) & 1 (ref) & 1 (ref) \\
\hline Low & $1.095(0.376-3.188)$ & .. &.. & $0.422(0.106-1.689)$ & $0.828(0.211-3.251)$ & $2.622(0.651-10.554)$ & $4.914^{* *}(1.399-1$ \\
\hline
\end{tabular}

HDI - Human Development Index, POE - Point of Entry, SPAR - State Party Self-Assessment Annual Reporting All data are shown in odds ratio ( $95 \%$ CI). Significant level: $\mathrm{p}<0.1^{*}, \mathrm{p}<0.05^{* * * *}, \mathrm{p}<0.01^{* * * * *}$ 
The study results showed that countries with high ITV had a significantly higher risk of importing COVID-19 cases earlier and were at higher risk of having greater numbers of case growing weeks than low ITV countries. Countries with high amount of international travel had higher risks of importing COVID-19 cases, and the days of reporting the first domestic case was earlier in those countries. Not surprisingly, high ITV countries had a significantly higher risk of having greater numbers of case growing weeks between Jan. 1 and the end of May because of their early importation of the virus. This result reflects the lack of success of most countries' border policies to keep the virus out of their country, which led to community spread. The low POE scores might be the reason for this phenomenon, and additional focus on improving POE capacity is recommended.

Furthermore, after the virus moved into communities, countries with low HE and low HSP had a significantly higher risk of having greater percentage of case growing weeks. This result means countries with low HE and low health care capacities had limited abilities to control the further community spread of COVID-19. Although researchers had pointed out the importance of health care systems in the fight against COVID-19 from the beginning of the pandemic, concern has been focused on the capacity to treat patients. ${ }^{30}$ However, the contribution of comprehensive medical coverage, which diminishes the financial burdens of people suffering from COVID-19, comprehensive coverage of medical institutions, which provide easy access to healthcare, and digitisation of health information, which allows easy tracking of patients' travel and medical histories, are also important in combatting COVID-19.31,32

In addition to the health care system, we found that health workforce density was significantly associated with countries' case PH measure speeds, which indicates the importance of having a sufficient healthcare workforce in the rapid response to infectious disease control. However, previous research that predicts the demand for a global health workforce in 2030 found that low-income countries will face severe shortages of healthcare workers needed to provide basic health services. ${ }^{33}$ This shortage might lead to lower SPAR scores as HSP and human resources scores in the future for low- and middle-income countries, and it is necessary to invest in healthcare workers for preventing the future emergence of infectious diseases.

\section{LIMITATIONS}

There are several limitations of this study. First, we could not rule out the possibility of COVID-19 information blockades and controls by some countries; therefore, it is, possible that their capacities were overvalued, and their COVID-19 outbreak progress was underreported in the study. Second, countries' self-reported assessments were not validated, although the previous study shown that the information is generally valid. ${ }^{10}$ The quality of a country's response is far more complex and subtle than can be measured by delays. Third, the relationship could only be considered associative rather than causal because of the study's cross-sectional design. Fourth, we measured infectious disease control outcomes by the governmental responses and the outbreak progress within the country, and other indica- tors, such as prevalence, incidence, and mortality were not taken into account because of concerns about the complicity of these indicators. Fifth, we used the direct data pooling strategy due to the lack of harmonised data collection and synthesis. There is a need of harmonised data collection and synthesis for optimizing the data usability and create joint reactions. Sixth, we could not rule out the impact of other possible influencing factor like population density, SARS experience and the perceived individual freedom on the national COVID-19 outbreak control outcome.

\section{CONCLUSIONS}

We found that although countries with high SPAR scores implemented PH measures significantly earlier than countries with low SPAR scores in general, but the response speeds of countries with very high and high HDI statuses were very different. Additionally, countries with low SPAR scores as HSP and low HE were significantly higher risk of upscaling the outbreak in the community. Because communication between health professionals and political leaders is important in activating infectious disease response systems, we suggested the need to include an item related to it in the SPAR tool. Also, further study regarding the role of UHC in infectious disease control is recommended.

\section{ACKNOWLEDGEMENTS}

We thank professor Ting-Wu Chuang for his valuable recommendation for analyses.

\section{AVAILABILITY OF DATA AND MATERIALS}

All the data in this research were obtained from publicly available sources from Our World in Data (https://ourworldindata.org/coronavirus), the World Bank (https://data.worldbank.org/), and WHO (https://apps.who.int/gho/data/node.home).

\section{ETHICS APPROVAL AND CONSENT TO PARTICIPATE}

Not applicable.

\section{FUNDING}

This study is funded by the Ministry of Science and Technology, Taiwan with the funding number as MOST108-2410-H-038-013-MY3 (3-2).

\section{AUTHORSHIP CONTRIBUTIONS}

For this article, FJT conceived the study and participated in literature review, discussion and drafted the manuscript. CPL participated in data analyses, result writing and manuscript editing. BT participated in data collection, and discussion. All authors read and approved the final version of the manuscript. 


\section{COMPETING INTERESTS}

The authors completed the Unified Competing Interest form at www.icmje.org/coi_disclosure.pdf (available upon request from the corresponding author), and declare no conflicts of interest.

\section{CORRESPONDENCE TO}

Feng-Jen Tsai

Ph.D Program in Global Health and Health Security, Mas- ter Program in Global Health and Development, College of Public Health, Taipei Medical University, $250 \mathrm{Wu}-\mathrm{Hsing}$ Street, Taipei, 110 TAIWAN.

jeanfjtsai@tmu.edu.tw

Submitted: February 09, 2021 GMT, Accepted: February 22, 2021 GMT 


\section{REFERENCES}

1. Tabish SA. Recent trends in emerging infectious diseases. International journal of health sciences. 2009;3(2):V-VIII.

2. Jones KE, Patel NG, Levy MA, et al. Global trends in emerging infectious diseases. Nature. 2008;451(7181):990-993. doi:10.1038/nature06536

3. Fan VY, Jamison DT, Summers LH. Pandemic risk: How large are the expected losses? Bull World Health Organ. 2018;96(2):129-134. doi:10.2471/blt.17.199588

4. WHO. WHO Coronavirus Disease (COVID-19) Dashboard. Published 2020. Accessed October 5, 2020. https://covid19.who.int/

5. WHO Headquarters. International Health Regulations (2005). 3rd ed. WHO Library Cataloguingin-Publication Data: World Health Organization; 2016.

6. IHR. Checklist and indicators for monitoring progress in the development of IHR core capacities in States Parties. Published online 2010. Accessed May 12, 2020. https://www.who.int/ihr/checklist/en/

7. WHO. Strengthening health security by implementing the International Health Regulations (2005). Published online 2014. Accessed October 5, 2020. https://www.who.int/ihr/procedures/monitorin g/en/

8. Kandel N, Sreedharan R, Chungong S, et al. Joint external evaluation process: Bringing multiple sectors together for global health security. The Lancet Global Health. 2017;5(9):e857-e858. doi:10.1016/s2214-109 x(17)30264-4

9. WHO. State Party Self-Assessment Annual Reporting Tool-International Health Regulations (2005). World Health Organization; 2018.

10. Tsai FJ, Tipayamongkholgul M. Are countries' self-reported assessments of their capacity for infectious disease control reliable? Associations among countries' self-reported international health regulation 2005 capacity assessments and infectious disease control outcomes. BMC Public Health. 2020;20(1):1-9.

11. Kandel N, Chungong S, Omaar A, Xing J. Health security capacities in the context of COVID-19 outbreak: An analysis of International Health Regulations annual report data from 182 countries. The Lancet. 2020;395(10229):1047-1053. doi:10.1016/ s0140-6736(20)30553-5
12. Prevent Pandemic. Country preparedness and COVID-19. Published 2020. Accessed May 10, 2020. ht tps://preventepidemics.org/covid19/science/insights/ country-preparedness-and-covid-19/

13. OurWorldinData. Coronavirus (COVID-19) Cases. Published 2020. Accessed July 15, 2020. https://ourwo rldindata.org/covid-cases\#total-confirmed-cases-ho w-rapidly-have-they-increased-compared-to-other-c ountries

14. Alicandro G, Remuzzi G, La Vecchia C. Italy’s first wave of the COVID-19 pandemic has ended: No excess mortality in May, 2020. The Lancet. 2020;396(10253):e27-e28. doi:10.1016/s0140-6736(2 0)31865-1

15. WHO. E-SPAR State Party Annual Report.; 2019. Accessed June 24, 2020. https://extranet.who.int/e-sp $\underline{\operatorname{ar}}$

16. WHO. Archived: WHO Timeline - COVID-19. Published 2020. Accessed September 21, 2020. http s://www.who.int/news-room/detail/27-04-2020-whotimeline---covid-19

17. OurWorldinData. COVID-19: Government Response Stringency Index. Published 2020. Accessed July 14, 2020. https://ourworldindata.org/grapher/cov id-stringency-index?time=2020-01-22..latest

18. OurWorldinData. Week by week change in confirmed COVID-19 cases. Published 2020. Accessed July 14, 2020. https://ourworldindata.org/grapher/we ekly-growth-covid-cases?time=2020-04-03..latest

19. Kluge H, Martín-Moreno JM, Emiroglu N, et al. Strengthening global health security by embedding the International Health Regulations requirements into national health systems. BMJ Glob Health. 2018;3(Suppl 1):e000656. doi:10.1136/bmigh-2017-00 $\underline{0656}$

20. The World Bank. Health expenditure. Published 2020. Accessed June 24, 2020. https://data.worldban k.org/indicator/SH.XPD.CHEX.GD.ZS

21. UNDP. Human Development Reports.; 2020. Accessed June 28, 2020. http://www.hdr.undp.org/

22. World Population Review. Human Development Index (HDI) by Country 2020. Published 2020. Accessed June 12, 2020. https://worldpopulationrevie w.com/country-rankings/hdi-by-country 
23. WHO. Global Health Observatory data repository. Published 2016. Accessed June 28, 2020. http://apps.w ho.int/gho/data/node.home

24. The World Bank. International tourism, number of arrivals. Published 2020. Accessed July 4, 2020. http s://data.worldbank.org/indicator/ST.INT.ARVL

25. The anti-science leadership of Trump, Bolsonaro, and Putin led to the worst coronavirus outbreaks in the world. Business Insider. Published online May 2020. Accessed September 25, 2020. https://www.busi nessinsider.com/trump-putin-and-bolsonaro-anti-sci ence-leadership-worst-coronavirus-outbreaks-2020-5

26. Ignoring Science during a Pandemic Is Poor Leadership. Scientific American. Published online 2020. Accessed September 28, 2020. https://www.scie ntificamerican.com/article/ignoring-science-duringa-pandemic-is-poor-leadership/

27. Leadership in covid-19: Building public trust is key. Thebmjopinion. Published May 5, 2020. Accessed August 26, 2020. https://blogs.bmj.com/bmj/2020/05/ 05/leadership-in-covid-19-building-public-trust-is-k ey/

28. Blumenthal D, Fowler EJ, Abrams M, Collins SR. Covid-19 - Implications for the Health Care System. N Engl J Med. 2020;383(15):1483-1488. doi:10.1056/n ejmsb2021088
29. Our World in Data. Which countries have protected both health and the economy in the pandemic? Published 2020. Accessed September 16, 2020. https://ourworldindata.org/covid-health-econo my

30. The Lancet. COVID-19: Protecting health-care workers. The Lancet. 2020;395(10228):922. doi:10.101 6/s0140-6736(20)30644-9

31. National Health Insurance. National Health Insurance's Contribution in Combating COVID-19. Published 2020. Accessed October 13, 2020. https://w ww.nhi.gov.tw/Content List.aspx?n=9DDC114B14A0 D426\&topn=787128DAD5F71B1A

32. Han E, Chiou ST, McKee M, Legido-Quigley H. The resilience of Taiwan's health system to address the COVID-19 pandemic. EClinicalMedicine.

2020;24:100437. doi:10.1016/j.eclinm.2020.100437

33. Liu JX, Goryakin Y, Maeda A, Bruckner T, Scheffler R. Global health workforce labor market projections for 2030. Hum Resour Health. 2017;15(1). doi:10.1186/ $\underline{\text { s12960-017-0187-2 }}$ 